This document is published in:

International Journal of Humanoid Robotics (2011), 8(1), 27-46.

DOI: $10.1142 / S 0219843611002332$

(C) 2011 World Scientific Publishing Company

This work has been supported by the CAM Project S2009/DPI-1559/ ROBOCITY2030 II, developed by the research team RoboticsLab at the University Carlos III of Madrid. 


\title{
A MODEL-FREE APPROACH FOR ACCURATE JOINT MOTION CONTROL IN HUMANOID LOCOMOTION
}

\author{
VILLAGRA, Jorge. Centro de Robótica y Automática, CSIC. jorge.villagra@iai.csic.es \\ BALAGUER, Carlos. Departamento de Ingeniería de Sistemas y Automática, \\ Universidad Carlos III de Madrid, balaguer@ing.uc3m.es
}

\begin{abstract}
A rew model-free approach to precisely control humanoid robot joints is presented in this article. An input-output online identification procedure will permit to compensate neglected or uncertain dynamics, such as, on the one hand, transmission and compliance nonlinear effects, and, on the other hand, network transmission delays. Robustness to parameter variations will be analyzed and compared to other advanced PID-based controllers. Simulations will show that not only good tracking quality can be obtained with this novel technique, but also that it provides a very robust behavior to the closed-loop system. Furthermore, a locomotion task will be tested in a complete humanoid simulator to highlight the suitability of this control approach for such complex systems.
\end{abstract}

Keywords: Humanoid robots; model-free control; joint motion control.

\section{Introduction}

Each joint of a humanoid robot is subject to complex and varying loads when the robot moves, and particularly in locomotion tasks. These loads are mostly due to the effect of gravity on the robots mass, but also include centrifugal and Coriolis forces from the robots complex motion. In addition, joints may become loaded and unloaded, as the robot lifts and places its feet. These different and demanding conditions make the whole system very sensitive to friction, flexibility, and compliance effects, especially when a belt transmission and a Harmonic Drive reduction are between motor shaft and joint axis (as in our specific RH2 humanoid design ${ }^{1}$ ). Moreover, joints may have some compliance due to structural stiffness, clearances, and backlashes. $^{2}$

It is then challenging to design controllers that maintain a high level of tracking performance under the aforementioned range of load conditions.

Due to the critical importance of some specific joints in humanoid robots, precision requirements have to be merged with robustness to flexibility and friction effects. For this reason, all joints in our RH2 robot $^{3}$ have been conceived with an additional absolute encoder in the shaft joint output, which will complement the classical motor incremental encoder (Fig. 1 shows the physical location of these components). 


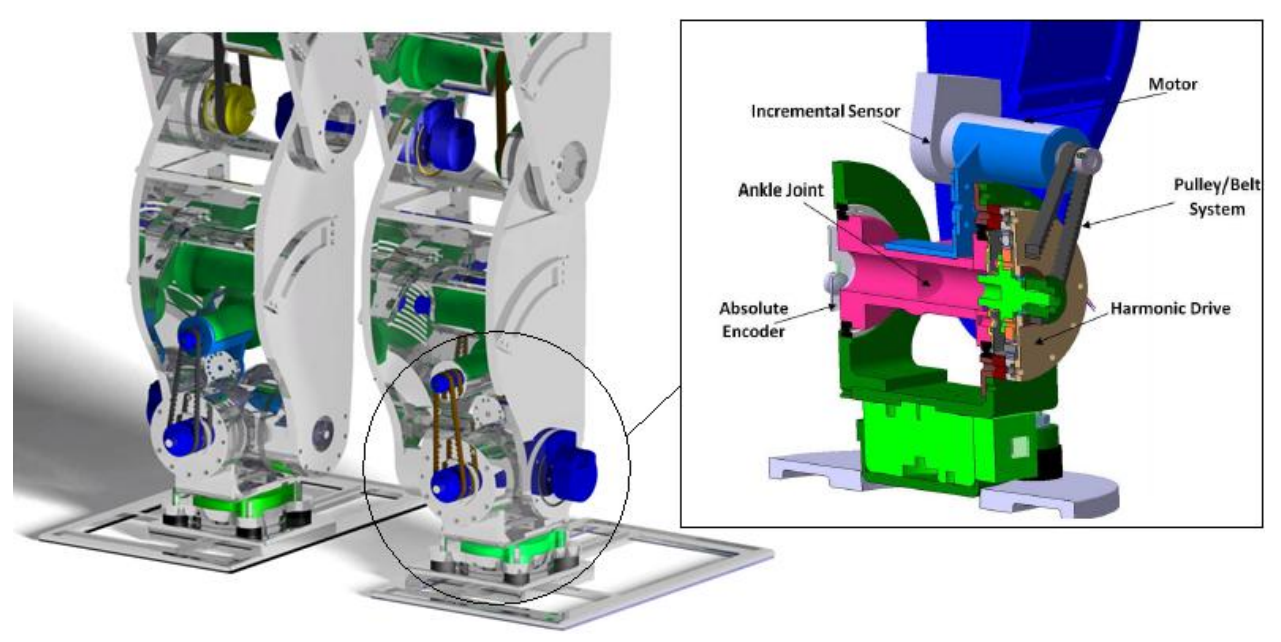

Fig. 1. RH-2 ankle design.

\subsection{Joint motion control under uncertainty}

The accurate position control of robot joints has been extensively studied in the last decades. Thus, many different feedback techniques have achieved good position tracking when considering electrically driven rigid robots. ${ }^{4}$ This task turns out to be critical in humanoid robots, since the stability margins must be respected as precisely as possible in a high range of speeds and under very different payload conditions. However, the transmission systems usually introduce nonlinear dynamics between the motor output and the real joint. Moreover, it is not easy to obtain a precise model of these effects, because of the great number of parts that intervene in the transmission.

Some authors ${ }^{5,6}$ have tried to obtain, through careful modeling, an accurate feedforward control which, in turn, allows to follow a desired trajectory with lowfeedback gains. The main problem of this sort of approaches is that they have to deal with highly cross-coupled nonlinear models, where the parameters are not always identifiable. Thus, several robust/intelligent control approaches ${ }^{7-10}$ and adaptive control techniques ${ }^{11-13}$ have been proposed to tackle with an appropriate feedback law the transmission uncertainty problem in rigid joint tracking. However, not so many efforts ${ }^{14}$ have been addressed to control electrically driven flexible-joints under uncertainty. This is because the presence of joint flexibility greatly increases the complexity of the system dynamics. ${ }^{a}$ In addition, the hardware architecture imposes absolute encoder information to pass through the controller area network (CAN) bus before coming back to the motor driver (Fig. 2). This message round-trip implies significant delays that make the analysis and control design more complex than with standard control techniques. 


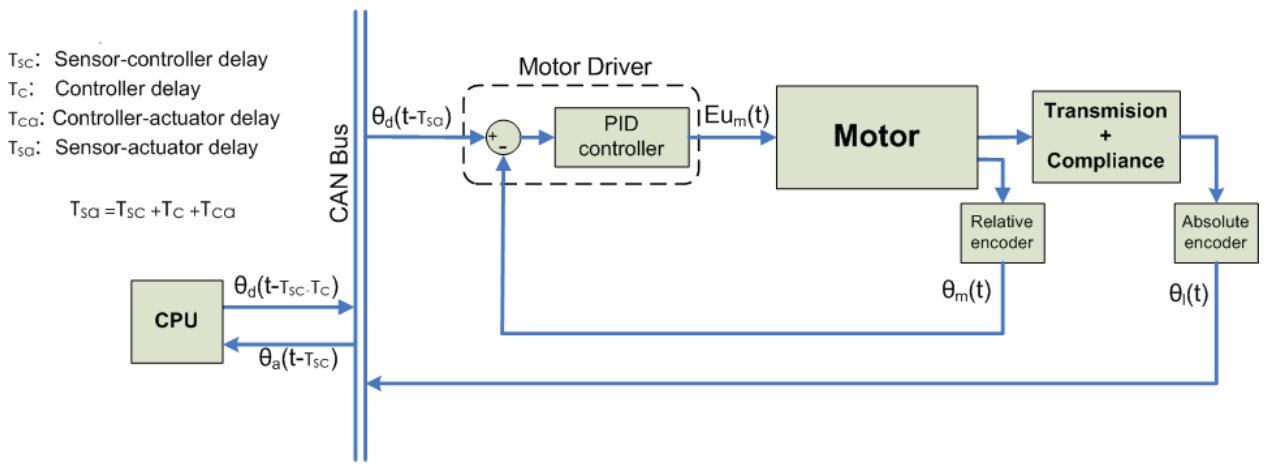

Fig. 2. Global joint control scheme.

Since the control algorithm has to be low-time consuming (and, therefore, easily implementable), any variation around PID controllers is an excellent candidate. Thanks to a novel model-free approach based on PID structure, transmission friction, uncertainty, and network delays will be fast and properly compensated. These techniques, initiated by Fliess, ${ }^{15}$ propose an algebraic framework to deal with fast numerical derivatives estimation, and thereafter, nonlinear model-free control design.

The rest of the article is organized as follows. Section 2 will be devoted to detail the system to be controlled. The model used in simulations will be composed of a DC motor, a Harmonic Drive transmission (modeled as a friction torque), and a flexible joint that reproduces the humanoid structure compliance. As depicted in Fig. 2, the outer loop control will be closed via the CAN bus, whose details in terms of transmission delay will be summarized also in Sec. 2. Since severe nonlinearities as well as complex time-varying phenomena appear in our system, an algebraic modelfree controller will be implemented. Its main features will be highlighted in Sec. 3. To show the advantages of the proposed control strategy, it will be compared in simulation with three other PID-based controllers in Sec. 5.1. Section 4 will be devoted to detail the benchmark and comment the results of this control law comparison. In Sec. 5.2, the proposed control technique is applied to each leg joint to show its suitability for locomotion tasks. Finally, some concluding remarks and prospective ideas will be given in Sec. 6 .

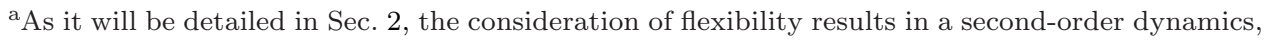
which, if the motor dynamics is also included, implies that a fifth-order dynamics should be considered.
} 


\section{System Model}

As presented in Fig. 1 and schematized in Fig. 2, the system to be controlled consist of a DC motor and a Harmonic Drive transmission system. Furthermore, flexion torques due to the robot structure compliance and Coulomb and viscous frictions of the Harmonic Drive have been taken into account.

\subsection{DC motor}

The classical direct current (DC) motor model is considered:

$$
\begin{aligned}
& J_{m} \ddot{\theta}_{m}(t)=K_{t} i(t)-\tau_{f r}(t)-\tau_{f l}(t) \\
& L_{m} \frac{d i(t)}{d t}=-R_{m} i(t)+E u_{m}(t)-K_{b} \dot{\theta}_{m}(t),
\end{aligned}
$$

where $i$ denotes the armature current, $\theta_{m}$ the angular position of the motor shaft, $J_{m}$ the rotor inertia, $L_{m}$ the terminal inductance, $R_{m}$ the motor terminal resistance, $K_{b}$ the back electromotive force constant, and $K_{b}$ the torque constant. $E$ represents the maximum available voltage, in absolute value, which excites the machine, while $u_{m}$ is an input voltage modulation signal, acting as the ultimate control input, with values restricted to the closed real set $[-1,1]$. Friction torque $\tau_{f r}$ generated by the Harmonic Drive transmission and flexible torque $\tau_{f l}$ due to the structure compliance will be above detailed.

\subsection{Harmonic drive transmission}

The model of the reducer can be written as follows ${ }^{16}$

$$
\dot{\theta}_{t}=\frac{\dot{\theta}_{m}}{N}, \quad \tau_{t}=\alpha_{m} N \tau_{m},
$$

where $\theta_{t}$ is the transmission angular position, $\tau_{t}$ is the transmission at the Harmonic Drive output, $N$ is the gearbox ratio, and $\alpha_{m}$ represents the torque transfer coefficient.

\subsection{Friction}

As for any mechanical systems, frictions are difficult to model and many works have been published on the subject either with a wide scope $^{6}$ or more focused in Harmonic Drive transmissions. ${ }^{5,16,17}$

Results from $\mathrm{Abba}^{16}$ will be used in this work due to the similar biped robot context for which that study was developed. Consider a velocity $\dot{\theta}$ and load $\tau_{l}$ 
dependent friction force

$$
\tau_{f r}=\left(F_{r}+\mu\left|\tau_{l}\right|\right)\left(1+f_{1} e^{-\left(\frac{\dot{\theta}}{\theta_{0}}\right)^{2}}\right) \operatorname{sign}(\dot{\theta})+f_{2} \dot{\theta}+f_{3} \dot{\theta}|\dot{\theta}|,
$$

where $F_{r}, \mu \theta_{0}, f_{1}, f_{2}$, and $f_{3}$ are experimentally obtained constant values.

The static friction is felt at the start but it is not very influent in general working conditions. Indeed, when the direction of the movement is reversed, the speed is canceled without relieving the materials so the Stribeck effect can be neglected $\left(f_{1}=0\right)$. In addition, the quadratic viscous friction effect appears little in the robot transmissions; therefore, the coefficient $f_{3}$ can be neglected. As a result, the previous friction torque can be written as $\tau_{f r}=\left(F_{r}+\mu|\Gamma|\right) \operatorname{sign}(\theta)+f_{2} \theta$. I t c a n $\mathrm{b}$ e $\operatorname{shown}^{16}$ that a precise model integrating nonsymmetrical terms of friction for the two directions of rotation can be obtained from the previous equation. Thus, the motor $\tau_{f r_{m}}$ and joint $\tau_{f r_{t}}$ friction torques (or in other words, the harmonic drive friction model) can be expressed as follows

$$
\begin{gathered}
\tau_{f r_{t}}(\dot{\theta})=\frac{C_{c_{1}}}{2} \operatorname{sign}\left(\dot{\theta}_{t}+1\right)+\frac{C_{c_{2}}}{2} \operatorname{sign}\left(\dot{\theta}_{t}-1\right)+f_{c} \dot{\theta}_{t} \\
\tau_{f r_{m}}\left(\dot{\theta}_{m}\right)=\frac{C_{m_{1}}}{2} \operatorname{sign}\left(\dot{\theta}_{m}+1\right)+\frac{C_{m_{2}}}{2} \operatorname{sign}\left(\dot{\theta}_{m}-1\right)+f_{m} \dot{\theta}_{m},
\end{gathered}
$$

with $C_{c_{1}}$ and $C_{c_{2}}$ terms of Coulomb friction in direct and opposite directions for the connection of the axis of the leg, $C_{m_{1}}$ and $C_{m_{2}}$ similar terms for the motor side, and $\left(f_{t}, f_{m}\right)$ transmission and motor viscous friction constants. Finally, if the motor-joint torque transmission relationship (2) is introduced in Eqs. (4) a n d ( 5), a single expression can be used to express overall friction effects:

$$
\tau_{f r}=f_{t} \dot{\theta}_{m}+C_{t} \operatorname{sign}\left(\dot{\theta}_{m}\right)+\Delta C_{t}
$$

with

$$
\begin{aligned}
f_{t} & =f_{m}+\frac{f_{c}}{\alpha_{m} N^{2}} \\
C_{t} & =\frac{C_{m_{1}}+C_{m_{2}}}{2}+\frac{C_{c_{1}}+C_{c_{2}}}{2 \alpha_{m} N} \text { and } \Delta C_{t}=\frac{C_{m_{1}}-C_{m_{2}}}{2}+\frac{C_{c_{1}}-C_{c_{2}}}{2 \alpha_{m} N} .
\end{aligned}
$$

\subsection{Flexible joint}

The dynamic effects induced by the structure compliance has been characterized as a flexible link. In the last years, an important effort has been devoted to modeling and control flexible joints. ${ }^{14,18-20}$ However, since a realistic behavior needs complex partial derivative equations, an approximate dynamic model has been chosen in order to make identification and simulation easier.

Consider the dynamics of the flexible system as follows ${ }^{19}$

$$
J_{l} \ddot{\theta}_{l}+\tau_{l}-\tau_{f l}=0, \quad \tau_{f l}=c\left(\theta_{t}-\theta_{l}\right),
$$

where $J_{l}$ the joint inertia, $c$ the joint stiffness constant, $\tau_{l}$ the load torque, and the $\tau_{f l}$ the flexible torque. 


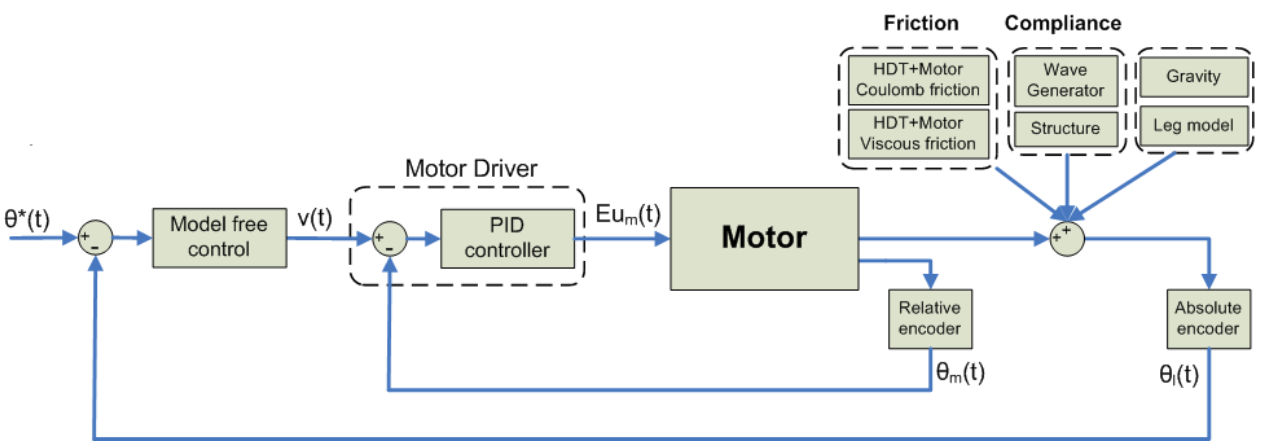

Fig. 3. Model-free joint control scheme in the absence of delays.

Remark that the torque $\tau_{f l}$, generated by joint flexible dynamics, is here the same than in Eq. (1), but with opposite sign.

\subsection{CAN bus}

As mentioned in Sec. 1, the absolute encoder located at the output shaft will provide, through a CAN bus, the necessary information to properly correct the joint reference from a global stabilizer.

As any other networked control system, the plant and the controller are spatially separated and the control loop is physically closed through the communication network. This wide spread approach in humanoid robotics results in decreased complexity and cost, easier maintenance and system diagnosis, and higher flexibility. However, the communication between the controller and the plant in such a way introduces a time delay into the closed-loop system. It is well known that time delay in a closed-control-loop degrades the performance and can lead to instability. Hence, with the aim of considering a model as realistic as possible, both sensorcontroller and controller-sensor transmission delays ( $T_{\mathrm{sc}}$ and $T_{\mathrm{cs}}$, respectively) have been taken into account in simulation. Furthermore, the CPU cycle time has been considered as an additional delay $T_{\text {ca }}$ (see Fig. 2).

\section{Model-Free Tracking}

In order to find out which is the reference angle $v(t)$ for the motor driver to get a better tracking at the output shaft, the following black-box closed-loop control scheme is proposed. ${ }^{21}$

Take a nonlinear finite-dimensional SISO system

$$
\Phi\left(t, \theta_{l}, \dot{\theta}_{l}, \ldots, \theta_{l}^{(\iota)}, v, \dot{v}, \ldots, v^{(\kappa)}\right)=0,
$$

where $\Phi$ is a sufficiently smooth function of its arguments. Assume that for some integer $n, 0<n \leq \iota, \frac{\partial \Phi}{\partial \theta_{l}^{(n)}} \not \equiv 0$. The implicit function theorem yields then locally

$$
\theta_{l}^{(n)}=\Upsilon\left(t, \theta_{l}, \dot{\theta}_{l}, \ldots, \theta_{l}^{(n-1)}, \theta_{l}^{(n+1)}, \ldots, \theta_{l}^{(\iota)}, v, \dot{v}, \ldots, v^{(\kappa)}\right) .
$$


This equation becomes by setting $\Upsilon=F+\alpha v$ :

$$
\theta_{l}^{(n)}=F+\alpha v,
$$

where $\alpha \in \mathbb{R}$ is a constant parameter, which is chosen in such a way that $F$ and $\alpha v$ are of the same magnitude and $F$ is determined thanks to the knowledge of $u, \alpha$, and of the estimate of $\theta_{l}^{(n)}$. It plays the role of a nonlinear back-box identifier.

If the simplest case is considered $(n=1)$, the term $F(t)$ reads at each sample time

$$
F\left(t_{k}\right)=\hat{\ddot{\theta}}_{l}\left(t_{k}\right)-\alpha v\left(t_{k-1}\right)
$$

The model locality implies that Eq. (8) is only valid in a very short period of time (one sampling period). If the sampling rate is high enough with respect to the system time constant, model (8) accurately represents the system dynamics, and, therefore, the desired behavior can be obtained by combining an inversion of Eq. (8) and a classical PID controller

$$
v(t)=\frac{1}{\alpha}\left(\dot{\theta}_{d}(t)-F(t)\right)+K_{P} e(t)+K_{I} \int e(t) d t+K_{D} \frac{d e(t)}{d t},
$$

where $\theta_{d}$ is a the reference trajectory coming from humanoid stabilizing control and inverse kinematics, $e=\theta_{l}-\theta_{d}$ is the tracking error, and $K_{P}, K_{I}, K_{D} \in \mathbb{R}$ are suitable gains.

The main advantage of this approach is that it uses a standard PID controller structure, but it is able to take into account, without any modeling procedure, the unknown parts of the system. Consequently, Fliess ${ }^{21}$ called this model-free technique intelligent PID (i-PID) control.

\subsection{Numerical differentiation}

This approach obviously necessitates robust derivatives estimation of noisy signals (see first term of Eq. (9)).

A vast literature exists on numerical derivative estimation. ${ }^{22}$ However, a very powerful technique, based on differential algebra and developed by Mboup, ${ }^{23}$ will be used in this work. Two main reasons explain this choice:

(1) They are algebraic and nonasymptotic, which results in a very fast estimation.

(2) They exhibit good robustness properties with respect to corrupting noises, without the need of knowing their statistical properties.

Using these techniques, the estimate of the first-order derivative of a noisy signal $\theta_{l}$ reads

$$
\hat{\dot{\theta}}_{l}(t)=\int_{0}^{T}(T-2 t) \theta_{l}(t) d t,
$$

where $[0, T]$ is a sliding time window. 
As it can be seen in Eq. (11), this approach needs the selection of one only parameter in the estimation procedure (window size $T$ ). At this respect, $\mathrm{Liu}^{24}$ has characterized the optimal window choice that minimizes the noise corruption for sinusoidal signals.

Remark 1. These differentiators are in general obtained via iterated time inte-grals of the noisy signal (in Eq. (11), one only integral is needed). Remark that time integrals are low pass filters that attenuate the corrupting noises. ${ }^{23}$

\section{A Control Law Comparison}

In order to show the efficiency of the proposed model-free tracking control, three different control algorithms will be compared. The joint output shaft will have to track a sinusoidal signal $\theta_{d}(t)$, whose frequency $(5 \mathrm{~Hz})$ is approximately equivalent to a $0.2-\mathrm{m} \cdot \mathrm{s}^{-1}$ walking motion. Even if support and swinging phases are completely different in terms of loads and joint speeds, a relatively high-frequency $(15 \mathrm{~Hz})$ and high-amplitude $(25 \mathrm{Nm})$ sinusoidal load $\tau_{l}(t)$ is considered all along the simulation. As a result, the control robustness to load variations can be evaluated under different situations.

All three strategies will be simulated on the above-presented model (Eqs. (1), (6), and (7)), with the parameter values summarized in Table 2 . These data come from an RE35-150W Maxon DC motor, friction parameters suggested by Abba, ${ }^{16}$ and inertial and stiffness parameters based on approximate structure mass distribution and compliance around critical joints. ${ }^{1,3}$ Besides, since CAN bus has a limited bandwidth $\left(1 \mathrm{MB} \cdot \mathrm{s}^{-1}\right)$, an approximate symmetric round trip time delay of $1.6 \mathrm{~ms}$ is considered (i.e. $T_{\mathrm{sc}}=0.8 \mathrm{~ms}, T_{\mathrm{ca}}=0.8 \mathrm{~ms}$ ). Additionally, the computer cycle time to generate joint set-points is fixed at $2.4 \mathrm{~m} \mathrm{~s}$.

Figure $4(\mathrm{a})$ shows the hardware architecture of the RH2 platform. Two CAN buses will be connected to a walking CPU, specifically dedicated to locomotion tasks, which will be in charge of controlling the 6 d.o.f. of each leg. This configuration will lead to an overall round-trip time delay of around $4 \mathrm{~ms}$ for each joint (see Fig. 4).

Finally, the absolute encoder is supposed to provide a perturbed signal, where the perturbation is modeled as an additive white Gaussian noise whose probability distribution is $\mathcal{N}(0, \sigma), \sigma=5 \cdot 10^{-5}$.

\subsection{Standard PID control}

The simplest control strategy that can be imagined in a robotic system is the one that control each joint axis as a SISO system in a decentralized way. Thus, coupling effects between joints due to varying configurations during motion are treated as disturbance inputs. Therefore, the structure of any controller should achieve an effective rejection of different disturbances appearing on the output shaft. Such constraint suggest the use of, on the one hand, an efficient feedforward control, and on the other hand, an integral action over the tracking error. 


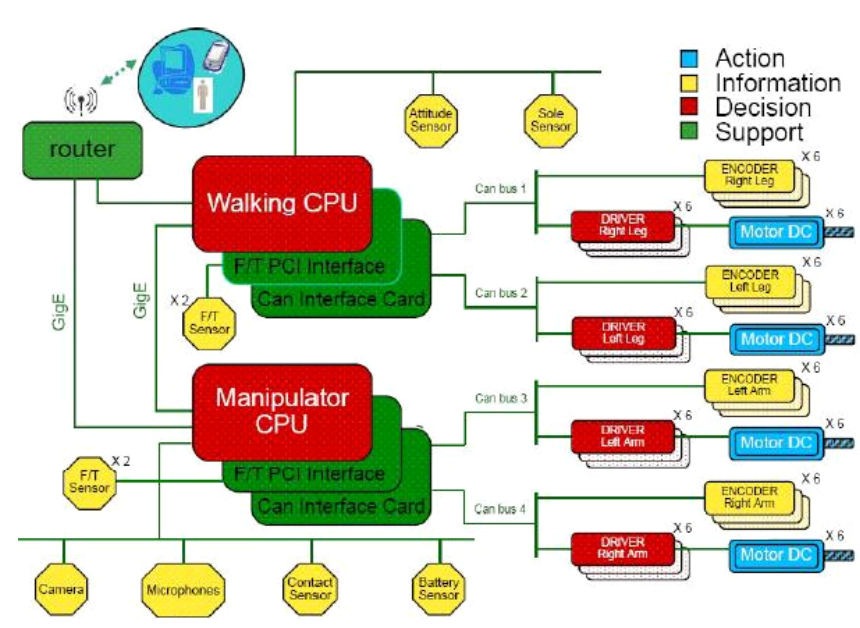

(a)

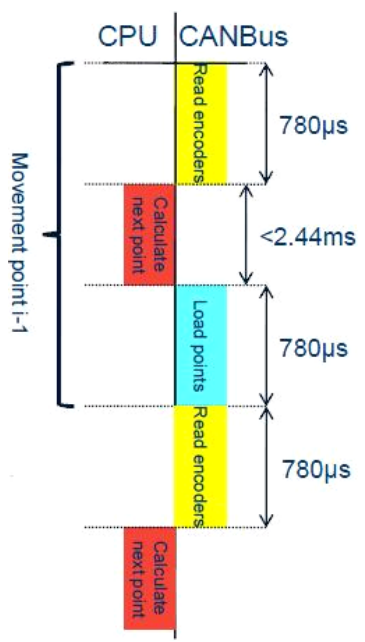

(b)

Fig. 4. (a) The RH-2 hardware architecture. (b) The round-trip time delay for each joint control.

In this connection, Siciliano ${ }^{25}$ proposes the following position based controller ${ }^{\mathrm{b}}$ :

$$
u(t)=u_{f f}(t)+K_{P_{i}} e_{\theta_{m}}(t)+K_{I_{i}} \int e_{\theta_{m}}(t) d t+K_{D_{i}} \frac{d e_{\theta_{m}}}{d t},
$$

where $u_{f f}(t)=K_{b} \dot{\theta}_{d}\left(t-T_{\mathrm{ca}}\right)+\frac{R_{m} J_{m}}{K_{t}} \ddot{\theta}_{d}\left(t-T_{\mathrm{ca}}\right)$ is the feedforward control, $e_{\theta_{m}}(t)=$ $\theta_{m}\left(t-T_{\mathrm{ca}}\right)-\theta_{d}\left(t-T_{\mathrm{ca}}\right)$ the angular position tracking error, and $K_{P_{i}}=R_{m}, K_{I_{i}}=$ $\omega^{2}{ }_{n}$ and $K_{D_{i}}=2 \eta \omega_{n}$ suitable PID gains. Note that these control parameters are optimally chosen ${ }^{25}$ in terms of the inner loop natural frequency $\omega_{n}$ and damping ratio $\eta$.

Remark 2. All continuous-time operators $\int d t$ and $d / d t$ are implemented on discrete time using its respective $z$-transforms.

\subsection{High level PID (HL-PID) control}

An outer PID control loop will generate appropriate set points for inner loop described above with absolute encoder information. Thus, the system governed by Eqs. (1), (6), (7), and (12) can be controlled by modifying the position reference, or in other words, by adding a new control variable $v(t)$. This outer control variable will have in this case the classical PID form

$$
\begin{aligned}
v(t) & =K_{P_{o}} e_{\theta_{l}}(t)+K_{I_{o}} \int e_{\theta_{l}}(t) d t+K_{D_{o}} \frac{d e_{\theta_{l}}(t)}{d t}, \\
e_{\theta_{l}}(t) & =\theta_{l}\left(t-T_{\mathrm{sc}}-T_{\mathrm{ca}}\right)-\theta_{d}\left(t-T_{\mathrm{ca}}\right) .
\end{aligned}
$$

${ }^{\mathrm{b}}$ This approach is equivalent to a position-velocity feedback, ${ }^{25}$ which is usually implemented on DC motor drivers. 


\subsection{PID with a feedforward term}

The structure of the controller should achieve an effective rejection of different disturbances appearing on the output shaft. Such constraint suggest the use of, on the one hand, an efficient feedforward control, and on the other hand, an integral action over the tracking error.

First of all, an outer PID control loop will generate appropriate setpoints for the inner loop with absolute encoder information. Thus, the system governed by Eqs. (1), (6), and (7) can be controlled by modifying the position reference, or in other words, by adding a new control variable $v(t)=\theta_{d}(t)$.

This outer control variable will have in this case the classical PID form

$$
\begin{aligned}
& v_{f b}(t)=K_{P} e_{\theta_{l}}(t)+K_{I} \int e_{\theta_{l}}(t) d t+K_{D} \frac{d e_{\theta_{l}}(t)}{d t} \\
& e_{\theta_{l}}(t)=\theta_{l}\left(t-T_{\mathrm{sc}}-T_{\mathrm{ca}}\right)-\theta_{d}\left(t-T_{\mathrm{ca}}\right) .
\end{aligned}
$$

The feedforward term could be obtained by combining transfer functions of the linearized model (friction is, therefore, neglected). Equations (1), (6), and (7) should then be properly manipulated and inverted to obtain the overall open-loop transfer function. However, in order to avoid the identification of every parameter, an inputoutput identification under nominal condition have been used. As a result, a secondorder system can replace the whole system, and therefore,

$$
v_{f f}(t)=a_{1} \hat{\theta}_{l}(t)+a_{2} \hat{\dot{\theta}}_{l}(t)+a_{3} \hat{\ddot{\theta}}_{l},
$$

where $a_{1}, a_{2}$, and $a_{3}$ are real scalar values and $\hat{\theta}_{a}, \hat{\dot{\theta}}_{a}$, and $\hat{\ddot{\theta}}_{a}$ are denoised estimates of the signal $\theta_{a}$ and its first and second derivatives.

The resultant control can be finally expressed as the sum of Eqs. (13) a n d (14)

$$
v(t)=v_{f f}(t)+v_{f b}(t) .
$$

\subsection{Model-free control}

HL-PID control will be here slightly modified to obtain an i-PID as in Eq. (10):

$$
\begin{aligned}
v\left(t_{k}\right) & =\frac{1}{\alpha}\left(\dot{\theta}_{d}\left(t_{k}\right)-F\left(t_{k}\right)\right)+K_{P} e_{\theta_{l}}\left(t_{k}\right)+K_{I} \int e_{\theta_{l}}\left(t_{k}\right) d t+K_{D} \frac{d e_{\theta_{l}}\left(t_{k}\right)}{d t}, \\
F\left(t_{k}\right) & =\hat{\dot{\theta}}_{l}\left(t_{k}\right)-\alpha v\left(t_{k-1}\right) .
\end{aligned}
$$

Note that Eq. (16) do not need any physical parameter from Eqs. (1)-(7). This fact turns out to be very useful to easily adapt the closed-loop system behavior to changing and unknown disturbances or parameter variations during the lifetime of the robot.

Remark 3. The incorporation of the time delay significantly complicates the model, as it changes from an ODE to a nonlinear infinite-dimensional system. As it will be shown in simulation, the i-PID ultra-local approximation will permit to 
control this complex system in a satisfactory way. However, this approximation is not necessarily valid to control any infinite-dimensional system and further research will have to be done to characterize the applicability domain of i-PID control.

\section{Simulation Results}

The robustness of the proposed control approaches will be thoroughly examined with the benchmark detailed in Sec. 4. Thereafter, a locomotion gait will computed to test in a humanoid simulator whether the joint motion i-PID control is suitable for this complex system.

\subsection{Joint control}

Control parameters have been chosen with the aim of minimizing the mean tracking error. To achieve such a task, the Matlab function fmincon (based on Sequential Quadratic Programming) has been applied to each control scheme.

Figure 5 shows simulation results for the above four approaches, from which several conclusions can be highlighted:

- Tracking quality of model-free algebraic control (i-PID) is considerably better than with the three other approaches (see mean and median tracking error in Table 1).

- However, it does not perform well enough during the initial transient. This fact has been already observed in other applications ${ }^{26,27}$ and is mainly due to the choice of $\alpha$. An easy way to overcome this problem is to let the control algorithm work for a short period of time before the desired maneuver starts.

- High-level PID (HL-PID) control and no absolute encoder based control provide very similar tracking behavior (in terms of maximum and mean error). This surprising phenomenon (HL-PID should a priori improve tracking performance) is mainly due to the feedback network delays (which do not exist in standard PID control). On the contrary, i-PID — and PID-FF to a slightly lesser extent — satisfactorily compensates these delays thanks to the ultra-local black-box identification.

- Control action is more jerky with HL-PID than with standard PID (see control FFT mean in Table 1). In any case, PID-FF and i-PID always provide softer control actions than the other two technique.

Even though, i-PID performs better than any of the presented PID-based control algorithms, the well-known PID-FF ${ }^{25}$ approach behaves is not far behind, especially in terms of control softness. However, the strong dependency on nominal parameters of the feedforward action motivates a parametric robustness comparison between i-PID and PID + FF.

Since no parameters explicitly appear in the closed-form i-PID controller, classical robust control tools cannot be exploited here to analyze the closed-loop system 


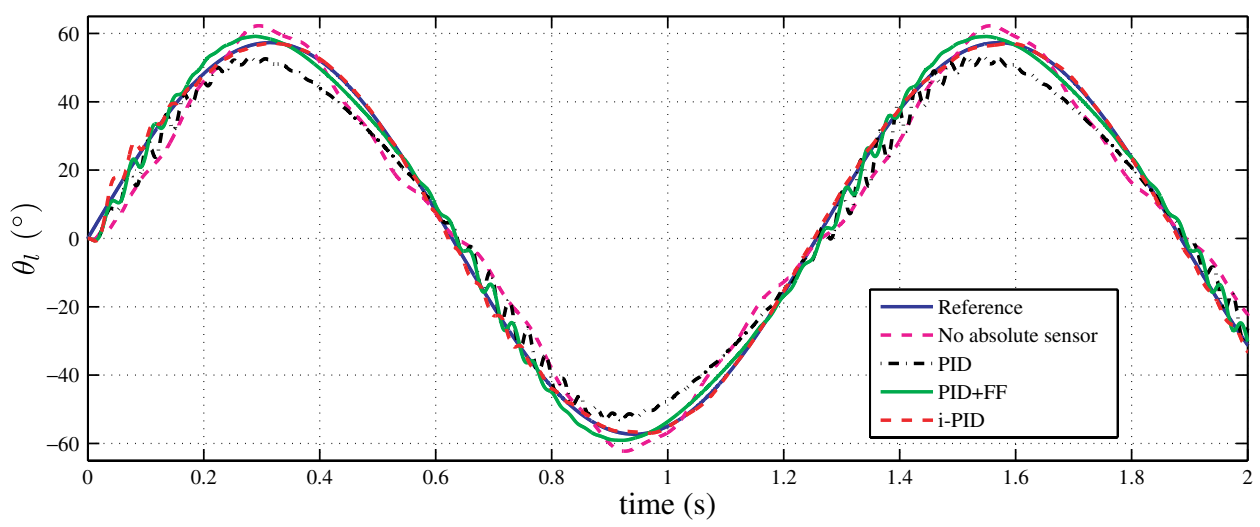

(a)

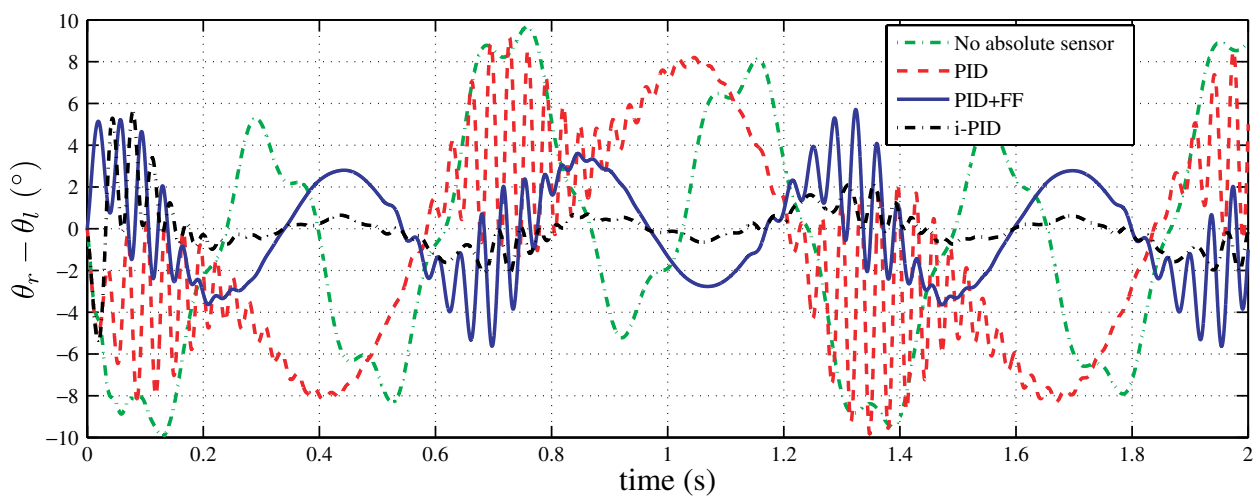

(b)

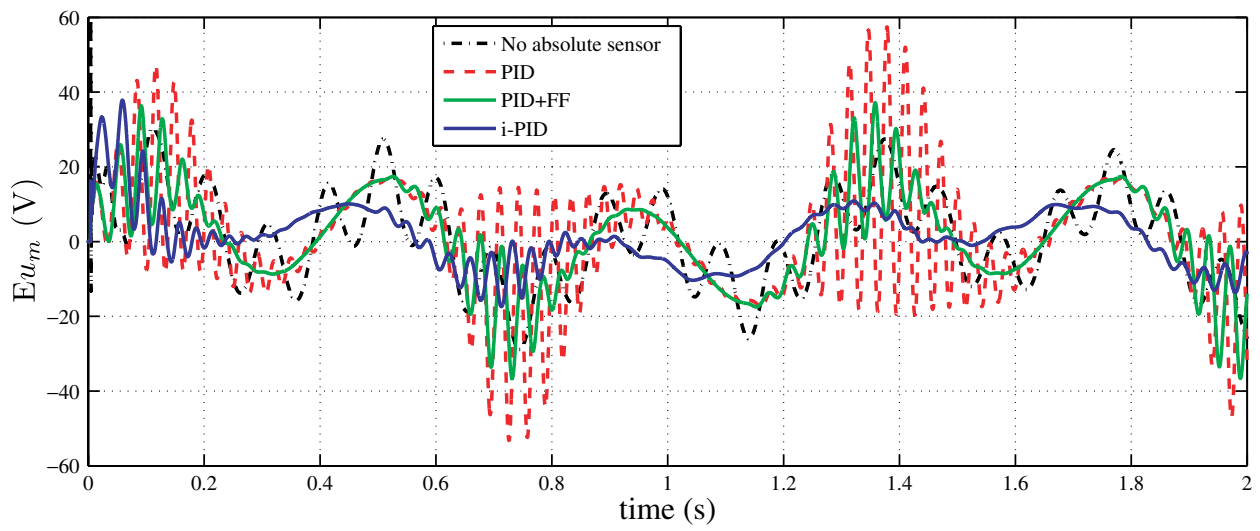

(c)

Fig. 5. (a) Position comparison, (b) position error comparison, and (c) control action comparison. 
Table 1. Tracking error and control FFT median of each control algorithm.

\begin{tabular}{lccc}
\hline Control Technique & $\begin{array}{c}\text { Mean Tracking } \\
\text { Error }(\mathrm{m})\end{array}$ & $\begin{array}{c}\text { Median Tracking } \\
\text { Error }(\mathrm{m})\end{array}$ & $\begin{array}{c}\text { Control FFT } \\
\text { Mean }(\mathrm{Hz})\end{array}$ \\
\hline No absolute sensor & 4.8369 & 4.8952 & 0.0156 \\
PID & 4.4293 & 4.5788 & 0.0181 \\
PID + feedforward & 2.0918 & 2.1181 & 0.0108 \\
i-PID & 0.7579 & 0.5134 & 0.0079 \\
\hline
\end{tabular}

Table 2. Parameter stable domain.

\begin{tabular}{lll}
\hline Parameter & Nominal Value $\left(p_{\text {nom }}\right)$ & Parameter Range \\
\hline Motor terminal resistance $\left(R_{m}\right)$ & $4.75 \Omega$ & {$[4.20,6.69]$} \\
Terminal inductance $\left(L_{m}\right)$ & $1.29 \cdot 10^{-3} \mathrm{H}$ & {$[1.04,4.74] \cdot 10^{-3}$} \\
Back EMF Constant $\left(K_{b}\right)$ & $0.0235 \mathrm{Vs} / \mathrm{Rad}$ & {$[0,13.76]$} \\
Torque Constant $\left(K_{t}\right)$ & $0.0758 \mathrm{Nm} / \mathrm{A}$ & {$[0.0476,9.7024]$} \\
Rotor Inertia $\left(J_{m}\right)$ & $6.52 \cdot 10^{-6} \mathrm{Kgm}^{2}$ & {$[1.26,19.4] \cdot 10^{-6}$} \\
Joint stiffness $(c)$ & $200 \mathrm{Nm} / \mathrm{rad}^{2}$ & {$[139,25600]$} \\
Joint inertia $\left(J_{t}\right)$ & $0.3 \mathrm{Kgm}^{2}$ & {$[0.234,0.398]$} \\
Sensor-controller delay $\left(T_{\mathrm{sc}}\right)$ & $0.002 \mathrm{~s}$ & {$[0,0.0028]$} \\
Controller-actuator delay $\left(T_{\mathrm{ca}}\right)$ & $0.004 \mathrm{~s}$ & {$[0,0.512]$} \\
Global Coulomb friction $\left(C_{t}\right)$ & $0.0203 \mathrm{Nm}$ & {$[0.0010,0.651]$} \\
Diff. of Coulomb friction $\left(\Delta C_{t}\right)$ & $-0.0025 \mathrm{Nm}$ & {$[0,-0.0324]$} \\
Global viscous friction $\left(f_{t}\right)$ & $6.5 \cdot 10^{-3} \mathrm{Nms} / \mathrm{rad}$ & {$\left[0,6.5 \cdot 10^{-3}\right]$} \\
Input amplitude $\left(A_{u}\right)$ & $1 \mathrm{rad}$ & {$[0,128]$} \\
Input frequency $\left(f_{u}\right)$ & $5 \mathrm{~s}^{-1}$ & {$[0,640]$} \\
Disturbance amplitude $\left(A_{d}\right)$ & $25 \mathrm{rad}$ & {$[0,3200]$} \\
Disturbance frequency $\left(f_{d}\right)$ & $15 \mathrm{~s}^{-1}$ & {$[0,1920]$} \\
\hline
\end{tabular}

sensitivity to disturbances or to parameter uncertainty. Therefore, nondeterministic techniques, and particularly Monte Carlo methods, seem to be the most adapted tool to prove robustness when an i-PID control law is used.

In this connection, a stable domain for every parameter has been independently computed $^{28}$ and it is shown in Table 2 .

To account for parametric uncertainty, we define the model's parameters as distributions of values rather than as single fixed values. We then perform a Monte Carlo simulation, running the model repeatedly with 1000 random combinations of parameter values. The first 12 parameters in Table 2 will be considered to follow cen-tered normal distributions of the form $\mathcal{N}\left(0, \sigma_{i}\right), \overline{3} \sigma_{i}={ }^{1} \min \left(\left|p_{\mathrm{nom}_{i}}-p_{\max _{i}}\right|, \mid\right.$ $\left.p_{\min _{i}} \mid\right)$. Amplitudes and frequencies of inputs and disturbances signals are defined asomtifiform distributions between their maximum ranges (see last four parameters in Table 2) to ensure that we cover the full design space.

Figure 6 shows histograms of mean tracking errors for PID + FF and i-PID after 1000 random simulations. Several conclusions can be highlighted from the analysis of these two charts.

- The number of valid samples (those that verify $J_{\text {tot }} \leq 0.2$ ) is $5 \%$ higher for i-PID than for PID-FF. Remark that these results are obtained within the most 


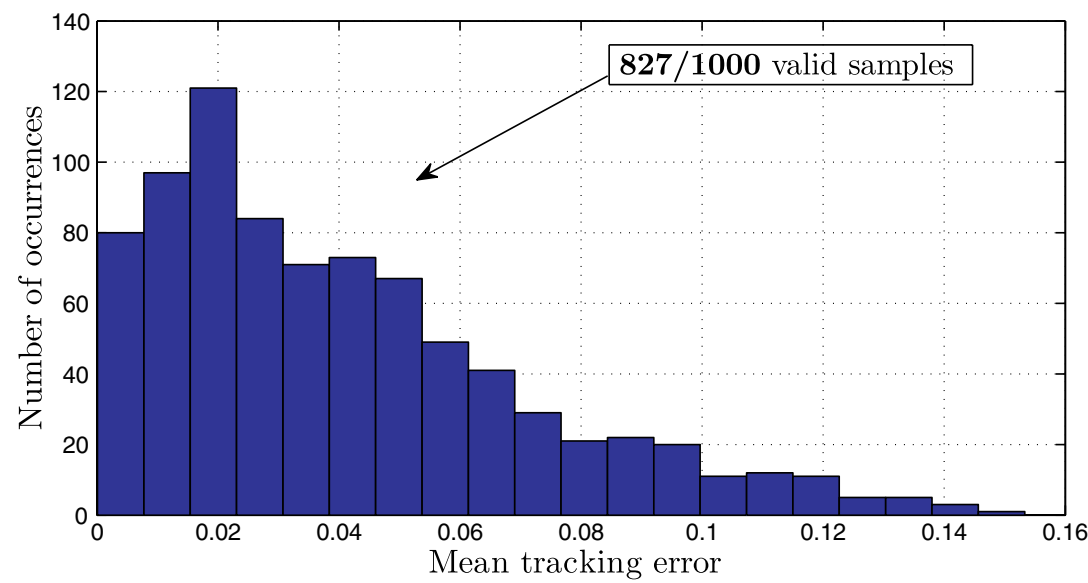

(a)

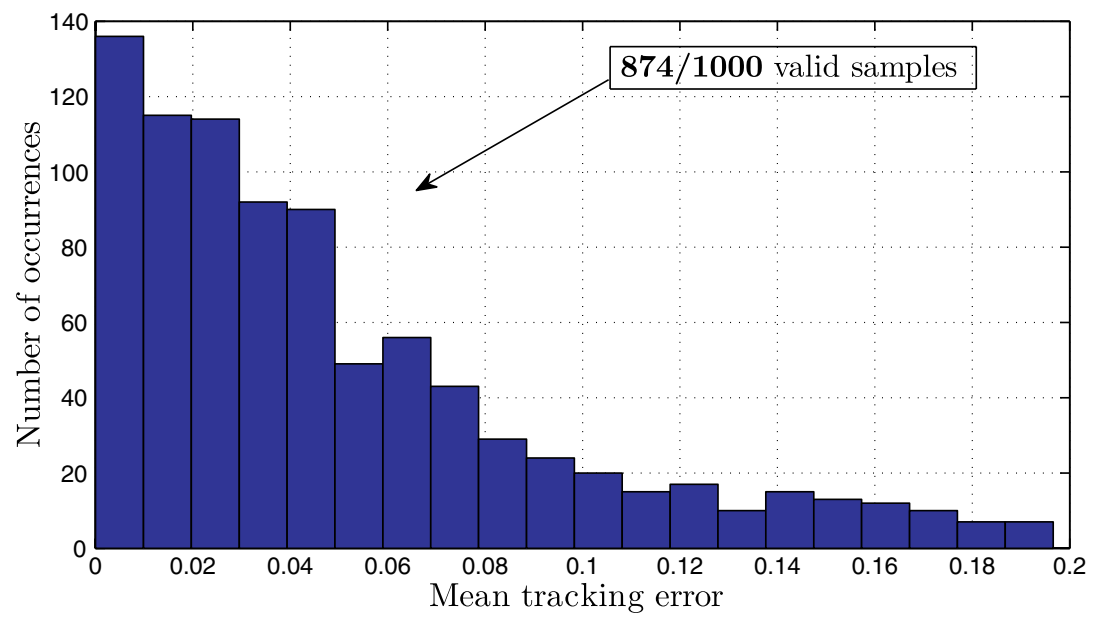

(b)

Fig. 6. Mean tracking error histogram for PID + FF (a) and i-PID (b).

conservative parameter domain, which is mainly constrained by PID-FF (see Table 2 ). It is then clear that if a broader parameter space had been used in Monte Carlo simulations, the difference between both methods would be even greater.

- A higher number of samples with low mean tracking error is obtained with i-PID than with PID-FF. This is consistent with the fact that i-PID generally provide a better tracking quality.

\subsection{Humanoid motion}

Once the performance of i-PID has been confronted with three other PID-based controllers to track a periodic trajectory under a high-frequency load disturbance, 


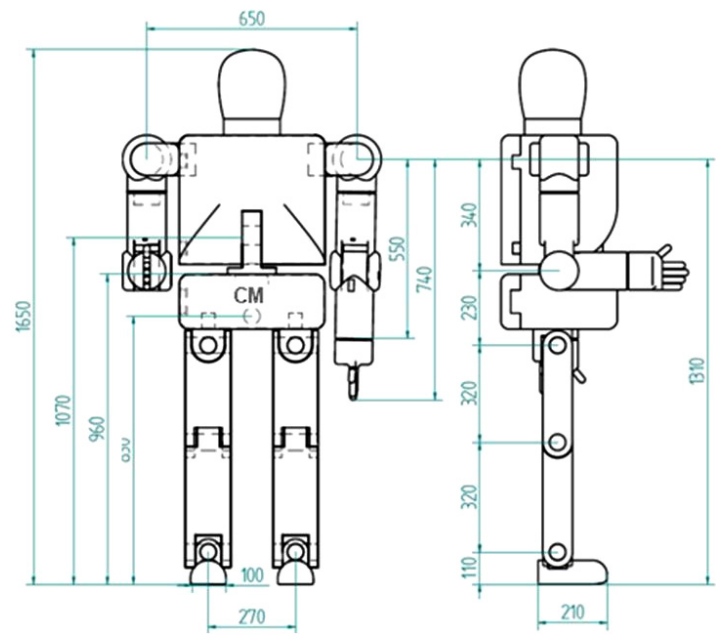

(a)

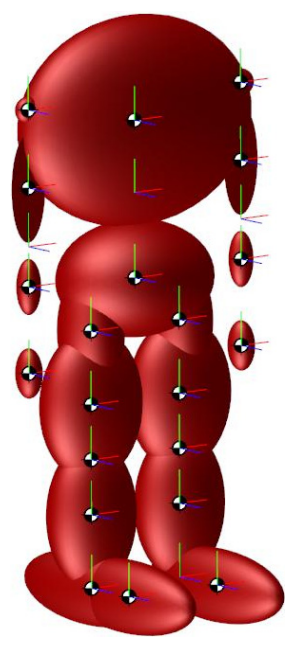

(b)

Fig. 7. (a) RH-2 design and (b) the simulated RH-2 in Simmechanics.

the behavior on a real humanoid platform — where every joint behavior is coupled with all the others - has to be tested.

To achieve such a task, a dynamic model using the Simmechanics Toolbox in Matlab (Fig. 7) has been developed. It represents the real humanoid platform RH-2, which is being designed and realized at Universidad Carlos III in Madrid (see 7 for the dimensions and the kinematical structure of the $\left.\operatorname{robot}^{1,3}\right)$.

This model introduces not only masses, inertias, and efforts, but also friction and compliance of each joint (as detailed in Secs. 2 and 5.1) of the robot. CAN bus roundtrip delays, measurement noises, and current, voltage, and torque limitations of the chosen actuators ${ }^{1}$ are also considered. Finally, the model also takes into account the floor reactions using a virtual spring-damper contact model. ${ }^{29}$

The influence of all the aforementioned effects will be tested with a specific humanoid movement. Since no stabilizer is still included in the simulator, and it is not easy to measure the influence of joint control in a complete gait cycle under these conditions, a stable acyclic gait ${ }^{30}$ has been used. This algorithm allow to generate a generic step from any configuration to other one. In this case, half of a gait cycle will be considered, i.e. a double-support phase and single-support phase (right-leg stance and left-leg swing). Thereafter, the inverse kinematics are computed, so that the joint motion control can be tested.

A stable swinging foot trajectories will be computed to simulate a step, whose length is $20 \mathrm{~cm}-x$ direction - and where the leg is lifted $4 \mathrm{~cm}-z$ direction during the floating phase. The total time of simulation has been chosen to be $t_{f}=0.9$ $\mathrm{s}$, with a swinging phase of $0.5 \mathrm{~s}$ (see Fig. 9), which, in a cyclic gait, would represent a $1.5-\mathrm{kmh}^{-1}$ walking speed. To show the performance of i-PID control, the resulting trajectories of three joints — left knee, ankle roll, and ankle pitch — are 

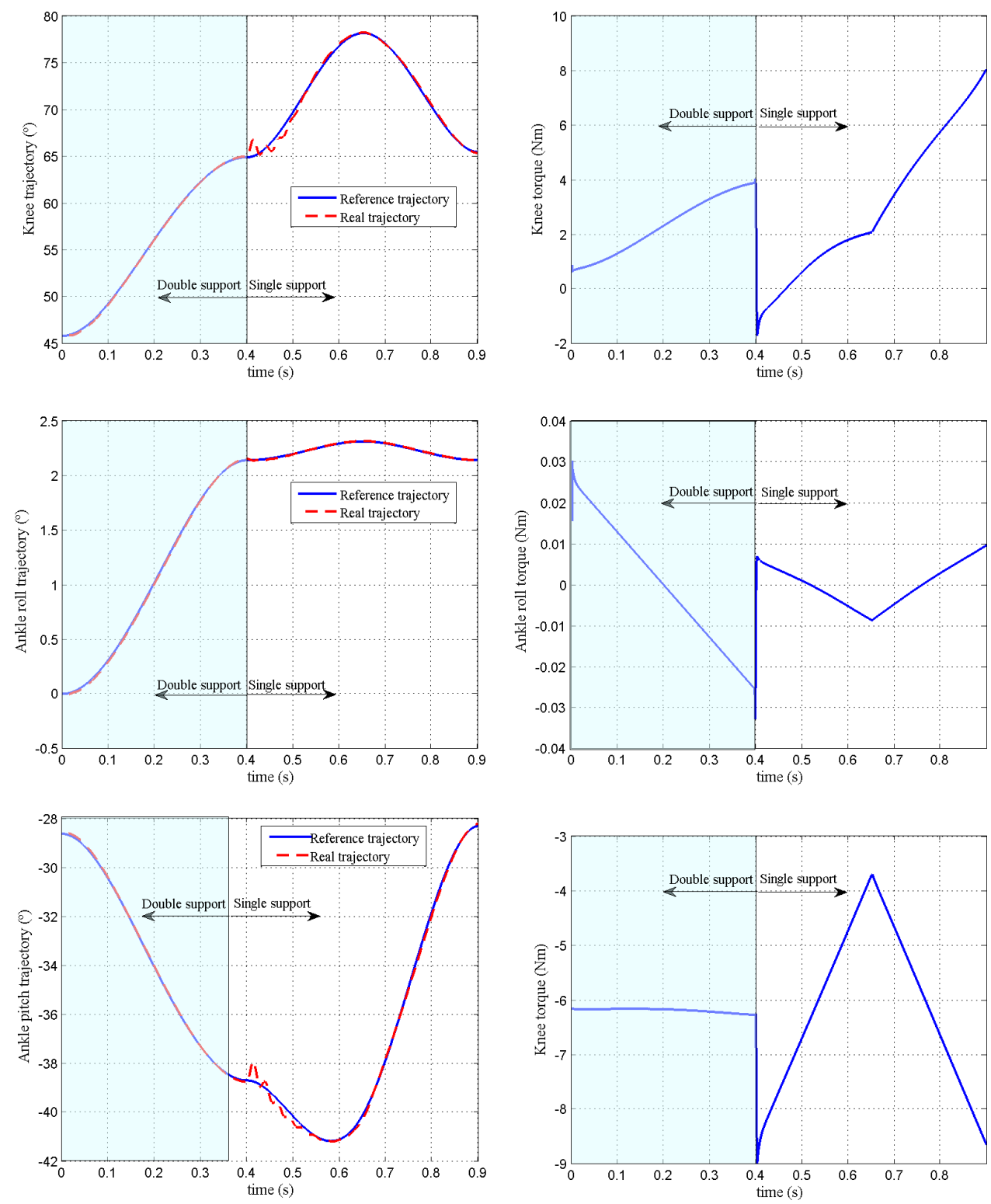

Fig. 8. Knee, ankle roll, and ankle pitch trajectories and loads.

plotted in Fig. 8. Remark that the main difficulty appears in the transition between double and single support, where floor reaction forces completely modify the loads distribution in robot floating leg. In any case, the tracking error never exceeds $1^{\circ}$ in the most delicate joints (pitch and roll ankle), and it is almost imperceptible in the knee. 


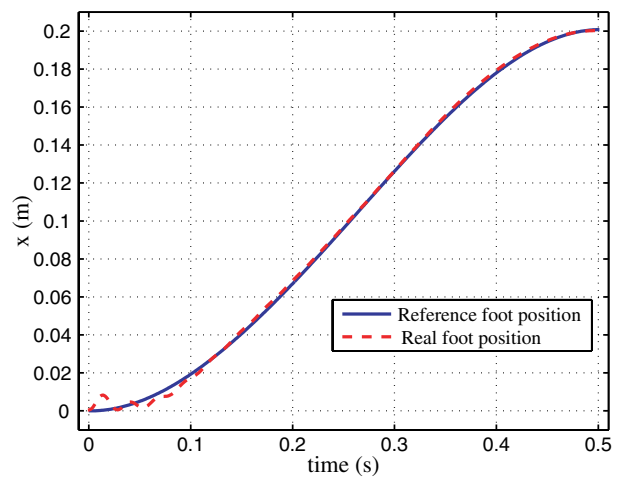

(a)
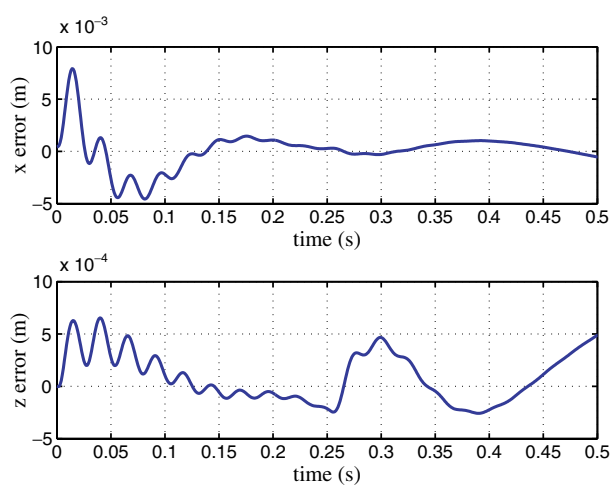

(c)

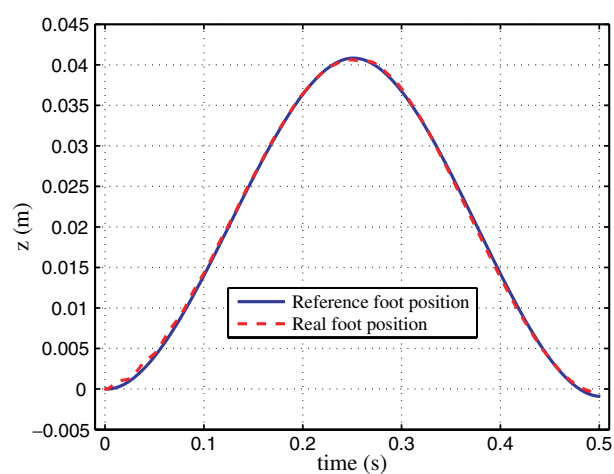

(b)

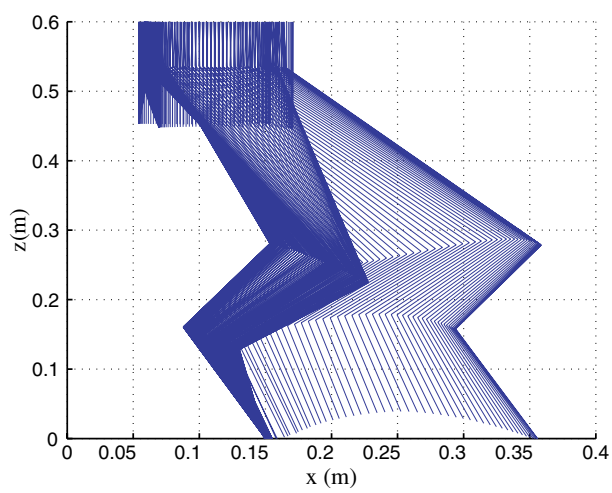

(d)

Fig. 9. (a) Left foot trajectory in $x$ direction, (b) left foot trajectory in $z$ direction, (c) foot tracking error, and (d) schematic representation of robot evolution.

The resulting floating foot trajectory is superimposed in $x$ and $z$ coordinates in Figs. 9(a) and 9(b), respectively. Besides a slight high-frequency oscillation at the beginning of the swinging phase, the acyclic gait is satisfactorily performed in this realistic simulation environment. Note in Fig. 9(c) that the foot follows the reference with a maximum error of $1 \mathrm{~cm}$ in each direction. As a result, the robot evolution perfectly fits the desired trajectory (see Fig. 9(d)).

\section{Concluding Remarks}

A new approach to precisely control humanoid robot joints has been presented. It is based on a novel model-free control technique (i-PID), which is able to compensate not only transmission and compliance nonlinear effects, but also CAN bus transmission delays. Simulations have shown that not only good tracking 
quality can be obtained with this i-PID, but also that it presents a remarkable robustness to nonlinear and poorly known effects and to extremely varying disturbances.

The promising preliminary results have been confirmed with more realistic trajectories and loads for a preplanned walking gait.

Finally, some experimental tests on a real platform will be carried out in order to validate the main features highlighted in this article.

\section{References}

1. L. Pabón, C. Pérez, J. Villagra and C. Balaguer, Mechatronic design and control of a critical biped robot joint, Proceedings of IEEE International Conference on Mechatronics (Malaga, Spain, 2009).

2. K. J. Lee, H .J. Yim, S. Jang, Y. S. Kang, Y. K. You and T. W. Park, A study on joint compliance for a biped robot, Proceedings of 5th IEEE-RAS International Conference on Humanoid Robots (Tsukuba, 2005).

3. C. Pérez, P. Pierro, S. Martínez de la Casa, L. Pabón, M. Arbulú and C. Balaguer, RH-2 an upgraded full-size humanoid platform, Proceedings of the 12th CLAWAR (Instanbul, Turkey, 2009).

4. T. J. Tarn, A. K. Bejczy, X. Yun and Z. Li, Effect of motor dynamics on nonlin-ear feedback robot arm control, IEEE Transaction on Robotics and Automation 7(1)(1991) $114-122$.

5. C. W. Kennedy and J. P. Desai, Modeling and control of the mitsubishi PA-10 robot arm harmonic drive system, IEEE/ASME Transactions on Mechatronics 10(3) (2005) 263-274.

6. T. Tjahjowidodo, F. Al-Bender, F. Van Brussel and W. Symens, Friction characterization and compensation in electromechanical systems, Journal of Sound and Vibration 308(3) (2007) 632-646.

7. B. S. Chen, H. J. Uang and C. S. Tseng, Robust tracking enhancement of robot systems including motor dynamics: A fuzzy-based dynamics game approach, IEEE Transactions on Fuzzy Systems 6(4) (1998) 538-552.

8. D. Kaynov and C. Balaguer, Joint control of a humanoid robot, Proceedings of 7 th IEEE-RAS International Conference on Humanoid Robots (Pittsburgh, 2007).

9. C. Kwan, F. L. Lewis and D. M. Dawson, Robust neural-network control of rigidlink electrically driven robots, IEEE Transactions on Neural Networks 9(4) (1998) $581-588$.

10. M. Oya, C. Y. Su and T. Kobayashi, State observer-based robust control scheme for electrically driven robot manipulators, IEEE Transactions on Robotics 20(4) (2004) 796-804.

11. Y. C. Chang, Adaptive tracking control for electrically-driven robots without overparametrization, International Journal of Adaptive Control and Signal Process 16(2) (2002) 123-150.

12. C. Ishii, T. Shen and Z. Qu, Lyapunov recursive design of robot adaptive tracking control with L2-gain performance for electrically-driven robot manipulators, International Journal of Control 74(8) (2001) 811-828.

13. C. Y. Su and Y. Stepanenko, Redesign of hybrid adaptive/robust motion control of rigid-link electrically-driven robot manipulators, Transactions on Robotics and Automation 14(4) (1998) 651-655. 
14. M. C. Chien and A. C. Huang, Adaptive control for flexible-joint electrically driven robot with time-varying uncertainties, IEEE Transactions on Industrial Electronics 54(2) (2007) 1032-1038.

15. M. Fliess, C. Join and H. Sira-Ramirez, Complex continuous nonlinear systems: Their black box identification and their control, Proceedings of 14 th IFAC Symposium on System Identification (Newcastle, Australia, 2006).

16. G. Abba and P. Sardain, Modeling of frictions in the transmission elements of a robot axis for its identification, Proceedings of the 16th IFAC World Congress (Prague, 2005).

17. R. Dhaouadi, F. H. Ghorbel and P. S. Gandhi, A new dynamic model of hystere-sis in harmonic drives, IEEE Transactions on Industrial Electronics 50(6) (2003) 1165-1171.

18. K. D. Dwidey and P. Eberhard, Dynamic analysis of flexible manipulators, a literature review, Mechanism and Machine Theory 41 (2006) 749-777.

19. F. Ghorbel, J. Y. Hung and M. W. Spong, Adaptive control of flexible-joint manipulators, Control Systems Magazine 9 (1989) 9-13.

20. S. Ozgoli and H. D. Taghirad, A survey on the control of flexible joint robots, Asian Journal of Control 8(4) (2006) 1-15.

21. M. Fliess and C. Join, Intelligent PID controllers, Proceedings of 16th Mediterrean Conference on Control and Automation (Ajaccio, France, 2008).

22. M. Fliess, C. Join and H. Sira-Ram'ırez, Non-linear estimation is easy, International Journal of Modelling Identification Control 4(1) (2008) 12-27.

23. M. Mboup, C. Join and M. Fliess, Numerical differentiation with annihilators in noisy environment, Numerical Algorithms 50 (2009) 439-467.

24. D. Liu, O. Gibaru, W. Perruquetti, M. Fliess and M. Mboup, An error analysis in the algebraic estimation of a noisy sinusoidal signal, Proceedings of 16th Mediterrean Conference on Control and Automation (Ajaccio, France, 2008).

25. B. Siciliano, L. Sciavicco, L. Villani and G. Oriolo, Robotics: Modelling, Planning and Control (Springer, 2009).

26. J. Villagra, D. Herrero and M. Abderrahim, Robust flatness-based control of an AGV under varying load and friction conditions, Proceedings of 7 th IEEE Conference on Control and Automation (Christchurch, New Zealand, 2009), pp. 1621-1628.

27. J. Villagra, B. Dandra-Novel, S. Choi, M. Fliess and H. Mounier, Robust stop and go control strategy: An algebraic approach for nonlinear estimation and control, International Journal of Vehicle Autonomous Systems 7(34) (2009) 270-291.

28. J. Villagra and C. Balaguer, Robust motion control for humanoid robot flexible joint, Proceedings of 18th Mediterranean Conference on Control and Automation (Marrakech, Morocco, 2010).

29. Y. Hwang, E. Inohira, A. Konno and M. Uchiyama, An order $n$ dynamic simulator for a humanoid robot with a virtual spring-damper contact model, Proceedings of IEEE International Conference on Robotics and Automation (Taipei, Taiwan, 2003).

30. P. Pierro, O. Stasse, A. Kheddar, K. Yokoi and C. Balaguer, Humanoid feet trajectory generation for the reduction of the dynamical effects, Proceedings of International Conference on Humanoid Robots (Paris, France, 2009).

31. M. Fliess and C. Join, Model-free control and intelligent PID controllers: Towards a possible trivialization of nonlinear control? Proceedings of 14th IFAC Symposium on System Identification (Saint Malo, France, 2009). 